\title{
ERRATA, VOLUME 76
}

Joan S. Birman, Abelian quotients of the mapping class group of a 2-manifold, pp. 147-150.

Page 149, equation (8): replace " $2 g+1$ " by " $2 g+2$."

Page 149, line 27: The commutator quotient group of $\mathrm{Sp}(2 g, z)$ is trivial if $g \geqq 3$ (Birman, On Siegel's modular group, Math. Ann. (to appear)). Thus our proof that $\left|A_{0}\right|=2$ if $g \geqq 3$ fails, and we can only say $\left|A_{\theta}\right|=1$ or 2 . We conjecture that the statement that $\left|A_{\theta}\right|=2$ is nevertheless true.

Robin Brooks, The number of roots of $f(x)=a, \mathrm{pp} .1050-1052$.

The second sentence of Theorem 2 on page 1051 is, in general, false; it is true when $Y$ is a compact orientable manifold.

\section{ERRATUM, VOLUME 77}

Gregers Krabbe, $A n$ algebra of generalized functions on an open interval; two-sided operational calculus, pp. 78-84.

On page 80 the line before Remark 3.1 reading tively, $\left.\left(1_{+} Q\right)\right),\left(1_{-}\right)^{2}=1=\left(1_{+}\right)^{2}$, and $\left(1_{-}\right)\left(1_{+}\right)=0$. should read:

tively, $\left.\left(1_{+} Q\right)\right),\left(1_{-}\right)^{2}=1_{-},\left(1_{+}\right)^{2}=1_{+}$, and $\left(1_{-}\right)\left(1_{+}\right)=0$. 